\title{
The Marine Realms Information Bank- A Coastal and Marine Digital Library
}

\section{What is the MRIB?}

The Marine Realms Information Bank (MRIB) is a digital library designed to classify, integrate, and facilitate access to scientific information about the oceans and the adjacent parts of the atmosphere and solid Earth, as well as to the people, techniques, and organizations involved in marine science. By integrating information science and communication technology, the MRIB creates a new vision of libraries and scientific publishing and provides a dynamic environment for the global sharing of digital information. The MRIB, which is a joint effort of the U.S. Geological Survey (USGS) and the Woods Hole Oceanographic Institution (WHOI), is available on the World Wide Web at http://mrib.usgs.gov.

\section{What is a Distributed Geolibrary?}

The MRIB is a distributed geolibrary as defined by the National Research Council (1999). As such, the MRIB (1) prioritizes the search and display of information by place (particular locations on the Earth's surface) and (2) links information existing in remote physical locations by removing geographic and temporal boundaries. In spite of this distributed nature, the basic services of the MRIB remain similar to those of a traditional library. Any piece of relevant information accessible online (text, pictures, charts, maps, or binary data files) can be classified by specialists and placed on virtual shelves designed to allow users to browse through holdings with shared subjects, locations, or other characteristics.

\section{How does the MRIB work?}

The conceptual design of the MRIB is that of a system capable of classifying and integrating existing information without altering its attributes or integrity. All information pertaining to coastal and marine environments is organized by the following 13 criteria: location, geologic time, physiographic feature, biota, disci-

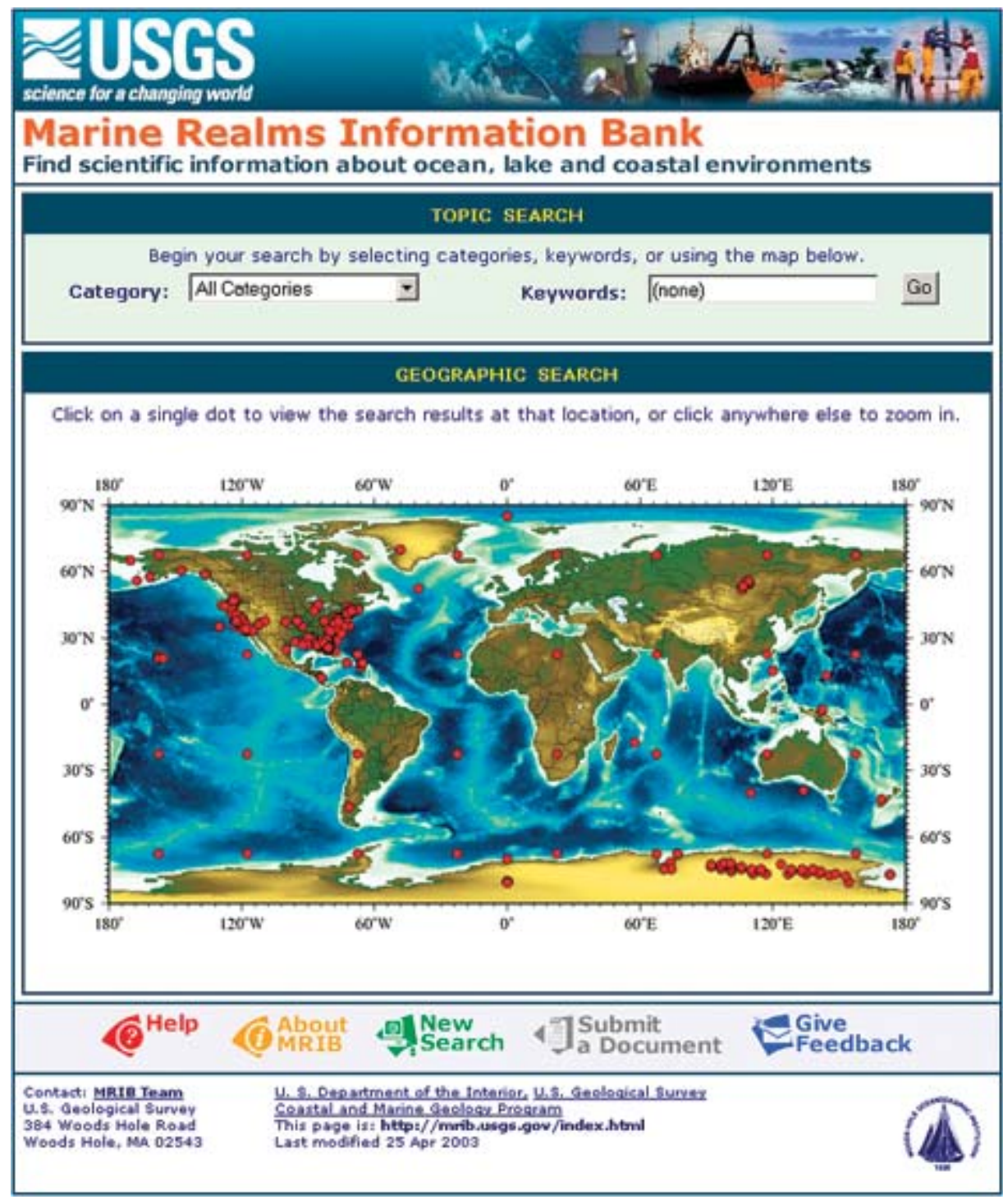

Figure 1. The M RIB Web interface.

pline, research method, hot topics, project title, agency name, author, class, format, and audience.

The collection of software constituting the MRIB grew out of the Woods Hole Oceanographic Institution's 4DGeoBrowser project (Lerner and Maffei, 2001), which is based on the electronic index card (EIC). An EIC is the electronic counterpart of the physical index card used in library card catalogs. Any digital information referenced in the MRIB is assigned an EIC containing descriptive metadata, including geospatial data (area of study) and the document's network location (Uniform Resource Locator or URL).

\section{What does an MRIB user see?}

The MRIB Web interface (fig. 1) is designed to encourage browsing as well as searching, thus preserving the strengths of a traditional library catalog while taking advantage of the flexibility of digital technology. Currently, the MRIB offers three types of searches-by category, by 


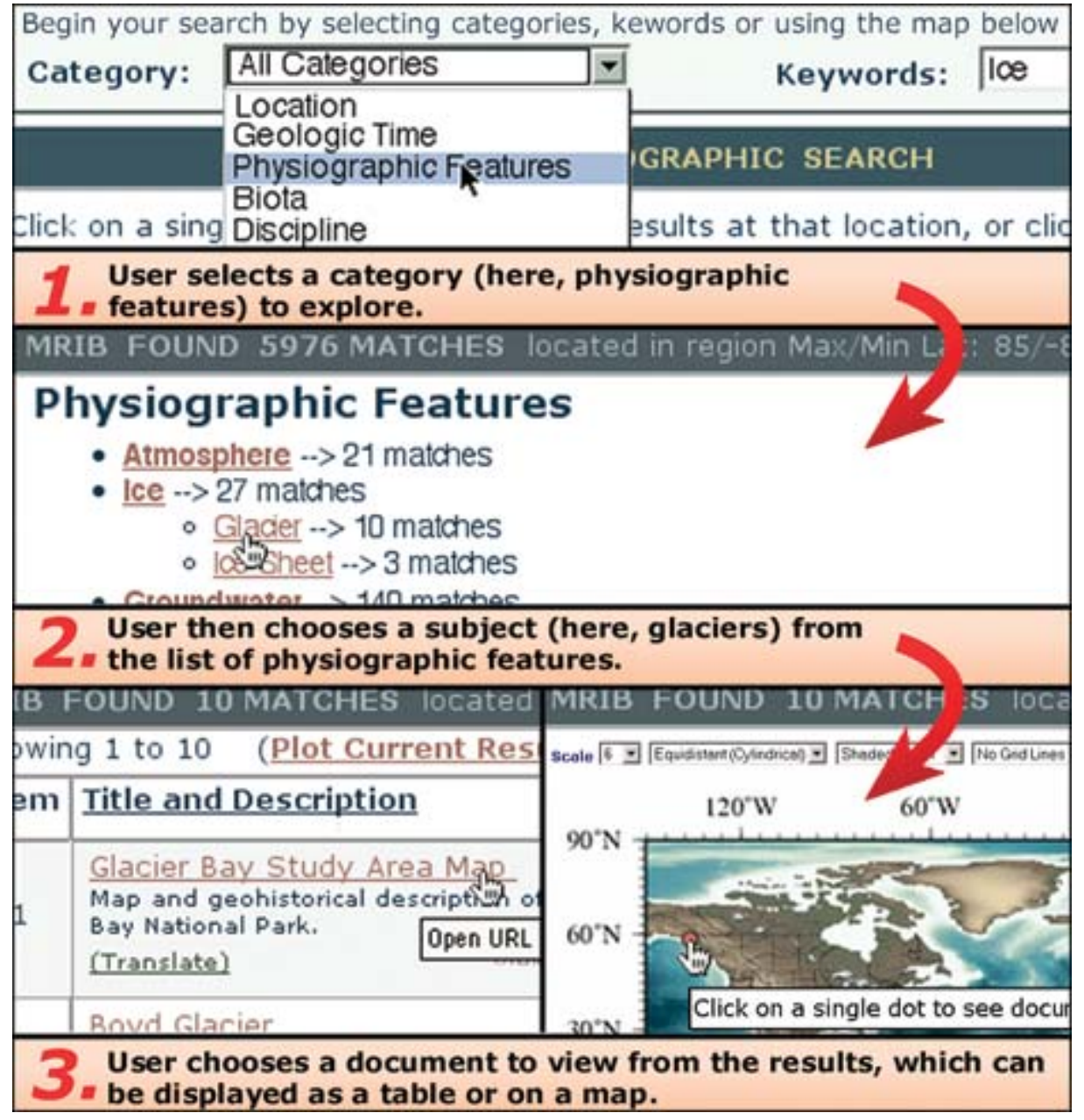

Figure 2. The M RIB Web interface is designed to encourage browsing as well as searching.

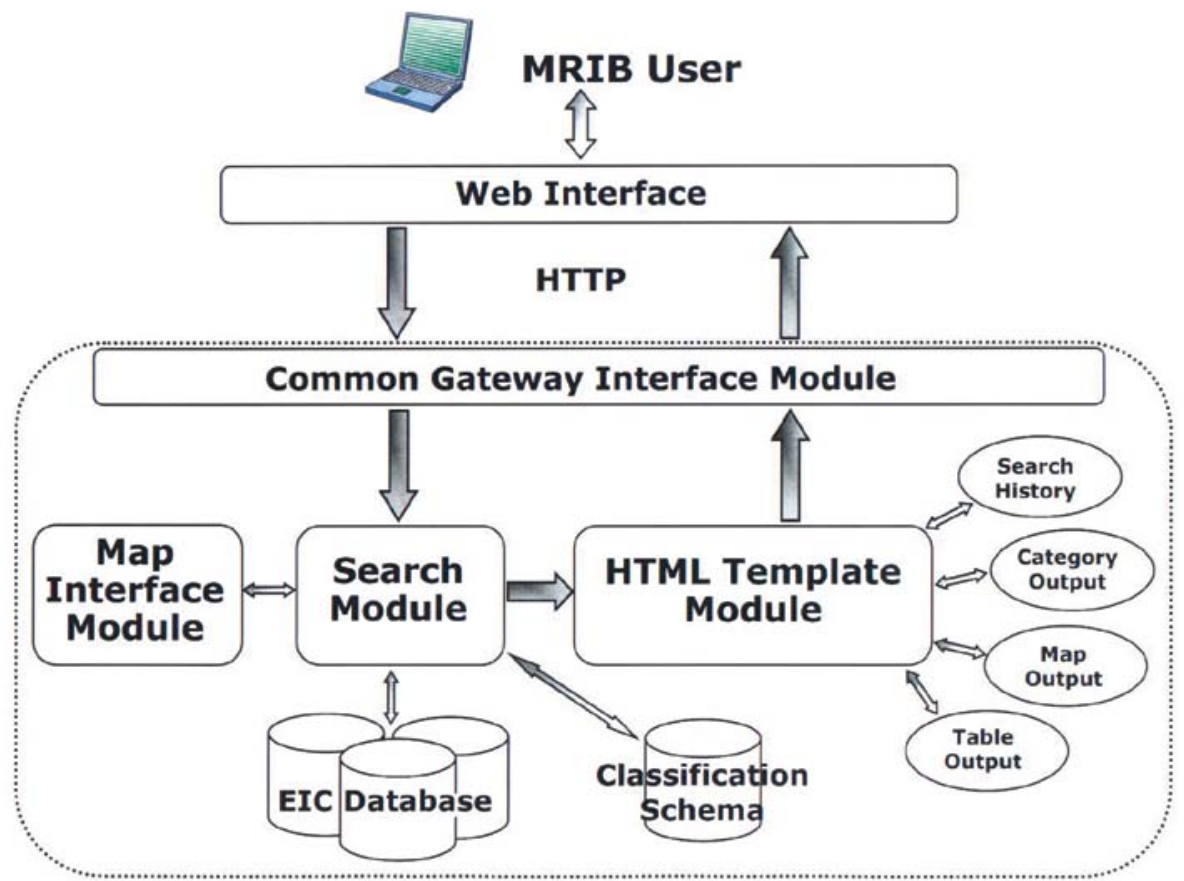

Figure 3. M RIB Software Architecture. The M RIB query process begins with the user issuing a search request on the Web brow ser. The Common Gatew ay Interface module parses this request and passes it on to the search module. The search module queries the electronic index card database for matching records; for geographical searches, the map interface module is also invoked to handle latitude and longitude parameters. Once the matching records are retrieved, the search module calls upon one of several templates chosen by the user (table, map, or category view ). Finally, the Web page of results is sent back to the user. keyword, and by location. Users can select categories from any depth within the classification hierarchies, search the metadata database for a keyword, or select a region by choosing a predefined location name listed in a gazetteer or by clicking on a map. These operations can be applied repeatedly, in any order, until the selection is appropriately focused. After each step, the MRIB displays maps and tables containing links and descriptions of the information resources matching the selection criteria (figs. 2 and 3).

\section{What is the importance of the M RIB?}

The initial focus of the MRIB has been on USGS coastal and marine geology information, but data from other scientific agencies, including WHOI, are also being indexed. MRIB Version 1 was released on January 2003. The significance of the MRIB project lies in the utility of the digital library itself and in the implementation of the distributed geolibraries concept. Systems similar to the MRIB can be used as unifying catalogs for extensive or rapidly developing information bases where a centralized repository is impractical. The MRIB is a timely endeavor that will aid in accessing scientific information and in understanding the complicated issues involved in managing natural resources and promoting public health, safety, and the well-being of our planet and its inhabitants.

\section{References Cited}

Lerner, S., and Maffei, A., 2001, 4DGeo-

Browser-A Web-based data browser and server for accessing and analyzing multi-disciplinary data: Woods Hole Oceanographic Institution, Woods Hole, MA, Technical Report WHOI-2001-13, $71 \mathrm{p}$.

National Research Council, 1999, Distributed geolibraries-Spatial information resources: National Academy Press, Washington, DC, $136 \mathrm{p}$.

\section{For additional information}

Frances L. Lightsom

U.S. Geological Survey

Woods Hole Science Center

384 Woods Hole Road

Woods Hole, MA 02540-1598

E-mail: flightsom@usgs.gov

Telephone: 508-457-2242

Fax: 508-457-2310 